MARTIN JÄGGLE*

\title{
INTERKULTURELLE KOMMUNIKATION
}

(Erhalten: 9 Oktober 2006; Angenommen: 18 Oktober 2006)

\begin{abstract}
Globalisierung, die es mit all ihren positiven wie negativen Seiten als ambivalentes Phänomen kritisch wahrzunehmen gilt, stellt zugleich Ursache und Kontext der Phänomene Interkulturalität und Transkulturalität dar. Die ,Verarbeitung“ der Globalisierung erfolgt jedoch immer lokal: diese Begegnungslinie zwischen dem Globalen und dem Lokalen wird auch als „Glokalisierung“ (glocalisation) bezeichnet. Auch dieser Begriff verweist darauf, dass eine neue, differenzierte Sichtweise von Kultur zu gewinnen ist. In der Zeit der kulturellen Pluralität erweist sich Interkulturalität nämlich als Normalfall, als ständiger Wechsel zwischen verschiedenen sozialen und kulturellen Identitäten, wobei kulturelle Verschiedenheit aufeinander verwiesen ist. Dementsprechend sind bei der Interkulturellen Kommunikation - verstanden als kommunikativer Aushandlungsprozess sozialer Deutungen - kulturelle Differenzen wahrzunehmen und anzuerkennen sowie kulturelle Zuschreibungen und Typisierungen eindeutig zu vermeiden. Interkulturelle Kompetenz gilt daher angesichts von Globalisierung, Migration, Pluralisierung der Lebensformen, Identitätsdiffusion und Risikogesellschaft als Schlüsselkompetenz, die allgemeine soziale und kommunikative Kompetenzen umfasst wie etwa die Fähigkeit zur Perspektivenübernahme und migrationsspezifisches Wissen.
\end{abstract}

Schlüsselbegriffe: Exklusion vs. Inklusion, Fremdheitserfahrung, Globalisierung, „Glokalisierung“, Interkulturalität, Interkulturelle Kompetenz, ,(inter)kulturelle Selbstreflexion“, Migration, „Transkulturalität“, Verstehen und seine Grenzen

Intercultural Communications: Globalisation - that has to be viewed critically as an ambivalent phenomenon with all its positive and negative features - is both a cause and a context of interculturality and transculturality. The effects of globalisation are, however, always elaborated at local levels: the encounter of the global and the local is often referred to as 'glocalisa-

\footnotetext{
* Martin Jäggle, Professur für Religionspädagogik und Katechetik an der Katholisch-Theologischen Fakultät, Universität Wien, Schenkenstr. 8-10, A-1010 Wien, Österreich; martin.jaeggle@univie.ac.at.
} 
tion'. This term is also indicative of the need for a new, more differentiated view of culture. In the era of cultural plurality, it is interculturality and the permanent change of various societal and cultural identities that can be regarded as normal phenomena; a process in which cultural diversity entails mutual interdependence. Accordingly, intercultural communication, in the sense of communicational conciliation along societal interpretations, is conditional upon the perception, acceptance and recognition of cultural differences, and the possible avoidance of cultural attributions and typifications. Therefore, in the light of globalisation, migration, pluralisation of styles of living, identity diffusion and risk society, intercultural competence is a key competence that implies both social and communicational competences, such as the acceptance of the perspective of others and migration-specific knowledge.

Keywords: exclusion vs. inclusion, experience of strangeness, globalisation, 'glocalisation', intercultural competence, '(inter)cultural self-reflection', interculturality, migration, 'transculturality', understanding and its limits

\section{Grundsätzliche Überlegungen}

\subsection{Vorbemerkung}

Wer sich auf die Frage der Interkulturellen Kommunikation einlässt, begibt sich auf Glatteis. Ist es möglich, den kulturellen Kontext von Kommunikation wahrzunehmen und persönliche, soziale sowie politische Quellen von „Kommunikationsstörungen“ dabei nicht aus den Augen zu verlieren, also der Kulturalismusfalle zu entgehen?

$\mathrm{Zu}$ viele wissenschaftliche Studien interpretieren Situationen, Verhaltensweisen und Einstellungen als kulturell bedingt und übersehen dabei die Bedeutung von Wohnund Einkommensverhältnissen, von sozialem Status und gesellschaftlicher Partizipation. Und doch ist im konkreten Leben und besonders in der Sozial-, Bildungs- und Gesundheitsarbeit den kulturellen Aspekten der Kommunikation verstärkt Aufmerksamkeit zu schenken. Das Problem der Interkulturellen Kommunikation wird im Bild vom Nachen auf hoher See treffend beschrieben, mit wenig Navigation und vielen Gefahren, zwischen Scylla und Charybdis, zwischen dem naiven Optimismus, "dass mit gutem Willen Interkulturelle Kommunikation funktioniert", wir sind doch alle Menschen, und dem Anspruch, ,dass man sich eben gut vorbereiten muss, und möglichst viel über andere Kulturen wissen sollte“. Und habe man dieses Abenteuer bestanden, sei „man noch nicht der Gefahr der Stereotypisierung entronnen“ (HERINGER 2004, 7).

Dem Kulturalismusvorwurf gilt es entgegen zu halten, dass jeder Mensch auf eine konkrete Sprache angewiesen ist, um sprechen zu lernen, um zugleich die Möglichkeit zu erwerben, auch andere Sprachen zu lernen. Es ist nicht möglich ,allgemein“ beziehungsweise ,universal“" sprechen zu lernen. Ebenso ist ein Mensch auf eine konkrete Kultur angewiesen, um in ihrem Kontext „kultiviert“ zu werden. Und so wie Sprache weder statisch noch eine Insel ist, sondern lebendig und voller ,fremder“ 
Einflüsse (Lehnworte, Fremdworte usw.), so ist auch das Bild von Kultur als einer Art Insel und der Mythos kultureller Reinheit realitätswidrig. Beides ist interessegeleitet, ein Mittel der Zuordnung und Ausgrenzung.

\subsection{Globalisierung als Ursache und Kontext}

Unser Thema muss - zumindest ansatzweise - in einem größeren Zusammenhang reflektiert werden, besonders im Horizont der Globalisierung, denn mit ihr ist etwa jene Migration verbunden, die (kulturelle) Differenz sichtbar macht, ohne sie allerdings allein zu verursachen. Interkulturelle Kommunikation und die Forderung nach Interkultureller Kompetenz sind nicht an die Anwesenheit von Migranten und Migrantinnen gebunden.

Globalisierung kann weder allgemein verbindlich definiert noch exakt beschrieben werden. Dass es um eine zunehmende wechselseitige Verknüpfung des Lebens der Menschen geht, sowohl des politischen, ökonomischen und sozialen Lebens, darüber könnte Einvernehmen erzielt werden. Je nachdem wie diese „,wechselseitigen Verknüpfungen" beurteilt werden, wird das Auftreten des Phänomens zeitlich verschieden angesetzt, vom ausgehenden 20. Jahrhundert bis zurück zum Beginn des interkulturellen Handels in der späten Bronzezeit.

Globalisierung kann als Phänomen der „Entgrenzung“ verstanden werden, der Veränderung, ja sogar der Aufhebung der Wirksamkeit räumlicher und zeitlicher Grenzen. In der Kategorie von Raum ist Globalisierung verbunden mit „Ausbreitung“ (BEYER 1994). Beyer sieht in der Globalisierung ein Phänomen des ausgehenden 20. Jahrhunderts, das er als Ausdehnung der Wirkung von westlicher Moderne auf die übrige Welt versteht. Zugleich kann Globalisierung verbunden werden mit „Verdichtung" (ROBERTSON 1992). Die technologische Revolution im Bereich der Kommunikationstechnologien hebt räumliche Distanzen auf. In der Kategorie von Zeit ist Globalisierung - als „Verdichtung“" verstanden - mit den Phänomenen der Gleichzeitigkeit und der (scheinbar unbegrenzten) Beschleunigung verbunden. Jeder Versuch, „der Vergangenheit Bedeutung beizumessen“ wird erschwert (SCHREITER 1997, 29).

Die „positiven“ Seiten der Globalisierung (,eine Welt", Kommunikation, globale Verantwortung) sollen nicht ausgeblendet, aber auch nicht gegengerechnet werden. Globalisierung ist ambivalent und es gilt daher auch, Globalisierung als ambivalentes Phänomen kritisch wahrzunehmen.

\section{3. „Glokalisierung“?}

Entgrenzung und Beschleunigung haben gravierende Folgen für alles spezifisch „Menschliche“: für das - einfache - Da-Sein, für Beziehung, Lachen und Weinen, Sinn, Kultur, Sprache, Kunst usw. Was eben an einen konkreten Raum, an Lokalität und eine konkrete und somit stets geschichtliche Zeit gebunden ist, wird ent-wertet. 
Zugleich aber wird Globalisierung auch lokal „verarbeitet“, leistet das Lokale Widerstand. Nach ROBERTSON (1998) lässt sich die Begegnungslinie zwischen dem Globalen und dem Lokalen als ,glocalisation“ („Glokalisierung“) bezeichnen. Der Neologismus, gebildet aus „globalisation“ und „locality“ wurde allerdings nicht von Robertson gebildet, aber durch ihn popularisiert. Bei allem Homogenisierungsdruck durch die Globalisierung würde das Lokale als bloß passives Element wahrscheinlich unzureichend wahrgenommen werden. Außerdem ist Raum nicht einfach Statik (und Zeit Dynamik), sondern ein komplexes Phänomen (Novy 2005). Durch eine neue, differenzierte Sichtweise des (lokalen) Raumes erhält Kultur eine neue Bedeutung.

Die Globalisierung erfordert „Kompetenzen für ein gelingendes Zusammenleben verschiedener kultureller, ethnischer und religiöser Traditionen und Identitäten“ (FRITSCH-OPPERMANN 2005, 19), wozu interkulturelle und interreligiöse Bildung erforderlich sind: „Bildung, wie wir sie brauchen, ist so gesehen nicht nur als Reaktion auf die Globalisierung zu verstehen, sondern auch als Initiative zu einer Globalisierung mit einem anderen Antlitz“ (Kirchenamt der EKD 2003, 77).

\subsection{Exklusion versus Inklusion}

Mit dem Prozess der Globalisierung eng verbunden ist die Exklusion von Einzelnen und Gruppen, von all jenen, die nicht mehr ,mitspielen“ können oder dürfen. In gewissem Sinne gefährdet Globalisierung Vielfalt und fördert zugleich Pluralität. Globalisierung macht jedenfalls Unterschiede (der Werte, des Lebenswissens, der Modelle guten Lebens, der Kulturen) sichtbar. Weder die euphorische Bejahung, noch die phobische Bekämpfung der Pluralität ermöglichen nun eine zukunftsfähige Identität. Im ersten Fall wird die Wahrheitsfrage und die kritische Auseinandersetzung ausgeklammert, droht Zynismus gegenüber den Opfern von Ideologien und Religionen. Im zweiten Fall bleibt nur Ausgrenzung, Bekämpfung, Sieg oder Niederlage, letztlich Krieg.

Laut UNDP findet Ausgrenzung aufgrund der Lebensweise dann statt, ,wenn der Kultur einer bestimmten ethnischen, rassischen oder religiösen Gruppe Anerkennung und Respekt verweigert werden" (NEIDLEIN 2004, 39). Ausgrenzung, also Exklusion wird sichtbar in unterschiedlichen Formen von Benachteiligungen in den verschiedenen gesellschaftlichen Bereichen nicht nur von Kultur, sondern auch von Wirtschaft und Politik. Da ein wesentlicher Teil von Kultur Sprache und Religion ist, trägt deren Nichtanerkennung maßgeblich zur Ausgrenzung bei.

Europa ist durch Gemeinsamkeiten in Geschichte und Kultur verbunden, die auch Grundlage für europäische Vielfalt geworden sind. Diese Gemeinsamkeit und Vielfalt wird in der (noch nicht ratifizierten) Verfassung der Europäischen Union anerkannt. Die Werte, auf denen sich die EU gründet, ,sind allen Mitgliedstaaten in einer Gesellschaft gemeinsam, die sich durch Pluralismus, Nichtdiskriminierung, Toleranz, Gerechtigkeit, Solidarität und die Gleichheit von Frauen und Männern auszeichnet" (Eine Verfassung für Europa 2005, I-2). Demnach würde dem Anspruch und der Realität Europas nur ein Inklusionskonzept entsprechen. 
Die humane Qualität einer Gesellschaft zeigt sich in ihrem Umgang mit Vielfalt. Die entsprechenden rechtlichen und politischen Regelungen sind ein Qualitätskriterium europäischer Demokratie. Dass mit Pluralität auch Konflikte verbunden sind, liegt nahe. Gesellschaftlich gesehen geht es dabei primär nicht um Konfliktvermeidung, sondern um einen angemessenen Umgang mit diesen Konflikten. Ein Aspekt sei hier nur angefragt: Ist die kulturelle Identität von Menschen, die stets prozessual konstituiert ist, nicht wesentlich von ihrer religiösen Orientierung mitbestimmt? Und sollten die religiösen Wurzeln besonderer kultureller Ausprägungen von Politik und Wirtschaft nicht verstärkt wahr- und ernst genommen werden?

Als Folge von Modernisierung, Migration, Globalisierung, Pluralisierung, et cetera kommt es zu einer wachsenden Differenzierung der Gesellschaft, die Individualisierung der Deutungsmuster unter anderen Gründen führen zu miteinander konkurrierenden unterschiedlichen Gesamtdeutungen und Gestaltungen von Wirklichkeit. Der Pluralismus wäre als Konkurrenz unterschiedlicher Kulturen zu verstehen. Mit der kulturellen Pluralität ist auch verbunden, dass die Einzelnen zugleich verschiedenen Gruppen und Kulturen angehören. So erweist sich Interkulturalität als Normalfall, als ständiger Wechsel zwischen verschiedenen sozialen und kulturellen Identitäten.

\subsection{Interkulturalität}

Interkulturalität geht von der Annahme aus, dass kulturelle Verschiedenheit aufeinander verwiesen ist, und stellt sich der Herausforderung, ein Miteinander zu verwirklichen in Respekt vor Eigenheit, Verschiedenheit und Fremdheit. Davon unterschieden ist Multikulturalität, die von der Annahme bestimmt ist, dass es eine Vielheit gibt, die keiner gemeinsamen Perspektive bedarf, sondern im Nebeneinander ihr Auskommen findet. Interkulturalität meint jene „Lebenswirklichkeit“, der sich die Menschen stellen müssen und die eine „kulturelle Überschneidungsituation“ darstellt. Nach Wierlacher stiftet sie gegenseitige Abhängigkeiten ,zwischen den agierenden Identitäten als Alteritäten, die für beide eine Veränderung ihrer selbst mit sich bringen kann" (HIERDEIS 2005, 128). Und Interkulturalität dient zugleich auch als Begriff in der wissenschaftlichen Auseinandersetzung mit dieser Lebenswirklichkeit.

\section{6. „Transkulturalität"“}

Die Einheit von Territorium und Kultur, also von Staat und Nation, erscheint zunehmend als fragwürdig. Mit Interkultureller Kommunikation bezeichnete etwa noch Gerhard MALETZKE (1966) den Prozess der Gedanken- und Bedeutungsvermittlung zwischen einzelnen Menschen oder Gruppen, die verschiedenen Kulturen angehören. Implizite Richtschnur bleibt dabei für Maletzke eine ,in sich homogene Nationalkultur, die sich territorial mit den jeweiligen Grenzen eines Staates deckt" (LÖFFELHOLZ \& HEPP 2002, 13-14). Transkulturelle Kommunikation hingegen zieht auch Konektivitäten „durch Kulturen hindurch“ in Betracht. Für Wolfgang Welsch geht der Begriff 
„Interkulturalität“ von „abgegrenzten und verschiedenen Kulturen aus“, um dann zu fragen, „wie Mitglieder dieser Kulturen miteinander kommunizieren, sich verstehen können“. Doch die Behauptung träfe heute nicht mehr zu, ,es gäbe territorial definierte (National-)Kulturen mit dem Nationalstaat als Grenze“. „Transkulturalität" will beides anzeigen: dass wir uns heute jenseits der klassischen Kulturverfassung befinden; und dass die neuen Kultur- und Lebensformen durch diese alten Formationen wie selbstverständlich hindurchgehen" (WELSCH 1992, 5). Die Lebensformen oder Lebensstile seien mit den herkömmlichen Kulturkategorien nicht mehr zu fassen. Daraus kann der Schluss gezogen werden: „Kultur und kulturelle Identität sind nicht (mehr) zwangsläufig territorial fixiert, sondern stehen in einem komplexen, widersprüchlichen und konfliktuären Prozess der mit der Globalisierung verbundenen Deterritorialisierung" (LÖFFELHOLZ \& HEPP 2002, 16). Welsch behauptet die Notwendigkeit einer „transversalen Vernunft“ zum Leben in der Vielfalt und sieht die Zukunft in der Transkulturalität und nicht in der Interkulturalität.

\section{Kommunikationstheorie von Watzlawick}

Für unsere spezielle Fragestellung lohnt es, die Kommunikationstheorie der Forschungsgruppe von Paul WATZLAWICK et al. (1969) heranzuziehen und sie auf ihre Relevanz zu befragen, nicht nur weil sie auch aus ethnographischem Fallmaterial entwickelt worden ist. Ihre fünf Axiome sind zu - sicher auch kritisierbaren - Standardthesen geworden:

\subsection{Axiom: „Man kann nicht nicht kommunizieren.“}

Sogar das als Nichtaktivität angesehene Schweigen ist in einen Kommunikationszusammenhang eingebettet und stellt daher eine Form der Kommunikation dar, ohne jeweils ,viel sagend“ zu sein. Die Bedeutung des Schweigens ist aber nicht nur von den Einzelnen und der jeweiligen konkreten Kommunikationssituation allein bestimmt, sondern auch vom kulturellen Kontext, innerhalb dessen kommuniziert wird beziehungsweise an dem die Einzelnen Anteil haben. Denn es gibt kulturell verschiedene Ausführungsbestimmungen, ,was als Schweigen zählt und als was Schweigen gilt“ (HERINGER 2004, 19).

\subsection{Axiom: Jede Kommunikation hat einen Inhalts- und einen Beziehungsaspekt.}

Die Beziehung legt letztlich die inhaltliche Bedeutung fest, weil sie bestimmt, wie Inhalte zu verstehen sind. Es gibt nun stärker sachorientierte Kulturen und stärker bezie- 
hungsorientierte Kulturen. Missverständnisse sind vorprogrammiert, wenn in einer Kommunikationssituation ein Partner aufgrund seiner Sachorientierung der Kultivierung der Beziehung eine zu geringe Bedeutung beimisst, was sein stärker beziehungsorientierter Partner sogar als persönlichen Affront erleben kann.

\subsection{Axiom: Interpunktion bedingt den Kommunikationsablauf.}

Es sind nicht Kausalketten, die den Ablauf einer Kommunikation und die Ursache von Kommunikationsstörungen sind, sondern die Interpunktionen. „Anfänge“ sind subjektiv gesetzte „Interpunktionen“. Klassisch ist die Studie von Margaret Mead über die Beziehung zwischen englischen Krankenschwestern und US-amerikanischen Soldaten während des Zweiten Weltkrieges geworden. Das Beispiel gibt wichtige Hinweise für Interkulturelle Kommunikation, auch wenn damals dieser Diskurshorizont in der heutigen Form nicht gegeben war. In der Abfolge von dreißig Schritten vom Beginn einer Bekanntschaft bis zum Geschlechtsverkehr stand Küssen in der US-amerikanischen „Flirt-Kultur“ an fünfter, in der englischen an fünfundzwanzigster Stelle. Somit galt ein US-amerikanischer Soldat als draufgängerisch, wenn er versuchte, eine englische Krankenschwester zu küssen, und ein englisches Mädchen als schamlos, wenn es nach der Zustimmung zum Küssen alsbald - ihrer kulturellen Norm entsprechend - bereits zum letzten Schritt bereit war.

\subsection{Axiom: Menschliche Kommunikation ist digital und analog.}

Digital ist Kommunikation, wo sie logisch, abstrakt ist, den Inhaltsaspekt repräsentiert, und analog ist sie, mit verbalen und nonverbalen Anteilen, wo sie sich besonders auf Beziehungen zwischen Dingen oder Menschen bezieht, wo sie Gefühle auszudrücken versucht und sich neben nonverbalem Ausdruck auch des Symbols als „Sprache des Möglichen“ sowie der Religion bedient. Je höher nun der Anteil der - auch kulturell geprägten - Bedeutungsvielfalt analoger Kommunikation ist, um so höher ist die Verstehensanforderung.

\subsection{Axiom: Kommunikation ist entweder symmetrisch oder komplementär.}

Kommunikation kann bestimmt sein vom Streben nach Gleichheit und Verminderung von Unterschieden zwischen den Partnern, sie kann aber auch bestimmt sein von gegenseitiger Ergänzung und somit vom Erhalt der Unterschiede. 


\title{
3. Vom Verstehen und seinen Grenzen
}

\subsection{Modell des Verstehens nach Frei}

Das Modell der wechselseitigen Wahrnehmung von zwei Personen nach Daniel Frei kennt vier unterschiedliche Dimensionen (HOLZBRECHER 1997, 161):

- das Selbstbild der Person A;

- das Fremdbild, das die Person A von der Person B hat;

- das Selbstbild, das die Person A der Person B unterstellt;

- das Fremdbild über die Person A, das die Person A der Person B unterstellt. Dasselbe gilt natürlich auch umgekehrt von der Person B.

Selbstbild und Fremdbild können hier als Konstruktionen betrachtet werden, deren beider Realitätsbezug erst aufzudecken wäre. Das Modell macht jedenfalls auf die enge Verflechtung von Selbstbild und Fremdbild aufmerksam sowie auf das Phänomen der Zuschreibung und der damit verbundenen Projektion. Die vom Modell nahegelegte praktische Konsequenz wäre die Forderung nach Empathie, also dem - einfühlenden - Versuch, die Welt mit den Augen des oder der Anderen zu sehen. Doch bleibt bei aller Möglichkeit und Berechtigung empathischer Übungen nicht eine - zu oft nicht bemerkte - unüberwindbare Grenze, da Menschen ja an die eigenen Verstehensvoraussetzungen gebunden sind? Oder wäre ein anderer Ansatz ergiebiger, nämlich jener der "Selbstaufklärung mit Hilfe der Augen der Anderen“ (SIMPFENDÖRFER 1981, 92)? Es sagt ja auch das, was Fremdes an Reaktionen auslöst, mehr über den einzelnen Menschen aus, als über Fremdes, das ja - empirisch feststellbar - als ambivalentes Phänomen ganz unterschiedliche Reaktionen auslöst.

Peukert gäbe hier zu denken: Descartes’ Versuch, „meine eigenen Gedanken zu reformieren und auf einem Boden zu bauen, der ganz mir gehört" ist im Prinzip

\begin{abstract}
die Methode der modernen empirischen Wissenschaften. Das entscheidende Problem ist dann jedoch, ob ich bei diesem Rückgang auf mein Konstruktionsvermögen auch die anderen mit meinen Mitteln glaube konstruieren zu können, oder ob ich den anderen als gleichermaßen frei und kreativ Konstruierenden anerkenne, der mir widersprechen kann, ob ich mich von ihm befragen lasse und in Frage stellen lasse und in einen Dialog eintrete.

(PEUKERT 2004, 372)
\end{abstract}

\subsection{Die Grenzen des Verstehens}

Verstehen hat eine Grenze, die in der konkreten Person, ihrer Einstellung und ihren Möglichkeiten begründet liegt. „Ich verstehe dich“ beendet einen Verstehensprozess. Dies kann unterschiedliche Bedeutung haben, Marianne Gronemeyer interpretiert es polemisch: „Ich bin mit dir fertig“ (HOLZBRECHER 1997, 153). Verstehen Wollen hat auch eine ethische Grenze, wenn es eine Form der Bemächtigung darstellt, also dem Machtgewinn über Andere dient. Außerdem gibt es ein Recht auf „Undurchsichtigkeit“. Nach Humboldt ist alles Verstehen „zugleich ein Nicht-Verstehen ... alle Über- 
einstimmung in Gedanken und Gefühlen zugleich ein Auseinandergehen" (PEUKERT 2004, 373). Borsche fasst die Gedanken von Humboldt so zusammen: „Verstehen überhaupt ist Anerkennen der Andersheit der Anderen“(PEUKERT 2004, 373).

\section{Theorie der Fremdheitserfahrung nach Schäffter}

\subsection{Einführung}

Nicht nur für Schäffter ist Fremdheit „keine Eigenschaft von Dingen oder Personen, sondern ein Beziehungsmodus“, wobei ,dessen Bedeutung sich nur dann voll erschließt, wenn man seine eigenen Anteile in diesem Beziehungsverhältnis mit zu berücksichtigen vermag“. Denn ,was ich und wie es als fremd erlebe“, hängt entscheidend von meiner (Lebens-)Geschichte ab. Die ,jeweilige personale und soziale Identität" ruft „,die Fremdartigkeit des Anderen“ hervor (SCHÄFFTER 1997, 3). Unsere Alltagserfahrung zeigt, wie unterschiedlich das Fremde wahrgenommen wird. Es wirkt bedrohlich und faszinierend, erscheint dunkel und hell, stößt auf Ablehnung und macht neugierig. Schäfter will den „Hintergrund des Spiegels“ ausleuchten, ,von dem es abhängt, welche Auffassungen von Fremdheit jeweils verfügbar werden“. Dafür unterscheidet er vier elementare „Ordnungsschemata systemspezifischer Innen/AußenBeziehungen“ und „die von ihnen konstituierten Modi des Fremderlebens“:

1. Ordnungen transzendenter Ganzheit: Das Fremde als tragender Grund und Resonanzboden von Eigenheit.

2. Ordnungen perfekter Vollkommenheit: Das Fremde als Negation von Eigenheit.

3. Ordnungskonzepte dynamischer Selbstveränderung: Fremdheit als Chance zur Ergänzung und Vervollständigung.

4. Konzeptionen komplementärer Ordnung: Eigenheit und Fremdheit als $\mathrm{Zu}-$ sammenspiel sich wechselseitig hervorrufender Kontrastierungen (SCHÄFFTER 1997, 7).

\subsection{Ad Modus 1}

Die „Deutung von Fremdheit als Entdeckung und Wiedergewinnung des eigenen Ursprungs“ lässt sich „als ein Rekurs auf die ,Conditio Humana“ begreifen“, beruhend ,auf der Prämisse einer grundsätzlichen Verstehbarkeit aller menschlichen Ausdrucksformen, sofern man nur selbst Zugang zur gemeinsamen anthropologischen Basis hat". Die Möglichkeit einer „Interkulturellen Hermeneutik“ stützt sich auf „existentielle transkulturelle Erfahrungen“" (SCHÄFFTER 1997, 10-11). 


\subsection{Ad Modus 2}

Wird „das Fremde zum Ausgegrenzten, das dem Eigenen ,wesensmäßig' nicht zugehörig ist“", kann es ,als Gegenbild gerade die Identität des Eigenen verstärken“, die innere Ordnung des Eigenen sichern. Das Fremde erscheint als bedrohlich, als feindlich, demgegenüber Abwehr und Abwertung notwendig ist. Aber die Abwertung des Fremden und die Selbstaufwertung bedingen einander. In der Dichotomie von Eigenheit und Fremdheit können die Vorzeichen auch wechseln und das Gegenbild wird zum Vor-Bild, das Fremde wird begehrenswert, weil dem Eigenen (kultur-)pessimistisch kein Wert mehr zugemessen wird (SCHÄFFTER 1997, 13-15). Für eine Interkulturelle Kommunikation bedeutet dieser Modus des Fremdverstehens verständlicherweise eine kaum zu überwindende Barriere.

\subsection{Ad Modus 3}

Angesichts steigender Komplexität bedarf es eines dynamischen Ordnungsgefüges. Darin erhält das Fremde „die Funktion eines externen Spielraums, der entwicklungsfördernde Impulse und strukturelle Lernanlässe erschließen hilft und in dem auch unvorhersehbare Entwicklungen möglich sind“. Mit der „Entdeckung bislang ungeahnter Möglichkeiten" werden Prozesse der Selbstveränderung freigesetzt. Fremderfahrung ermöglicht Selbsterfahrung. Es hängt aber auch von der ,inneren Integrationsfähigkeit“ ab, ob Selbstveränderung zu einer „Bereicherung“ oder zu einer „,systemsprengenden Überforderung" führt. Denn dieser Modus ist ebenfalls ambivalent. Einerseits kann die „Entdeckung von Fremdheit als Wiedergewinnung abgespaltener Erfahrungsmöglichkeiten und als Entfaltung latenter Potenzen der Eigenheit gedeutet werden", andererseits kann die „Akkomodation fremder Strukturen die interne Verarbeitungskapazität“ schwächen und wird „daher als Selbstentfremdung erlebt“" (SCHÄFFTER 1997, 16-19). Im letzteren Fall werde wohl aus Gründen der Sicherheit auf eine schroffe Abgrenzung zwischen Eigenem und Fremden (vgl. Modus 2) zurückgegriffen.

\subsection{Ad Modus 4}

Hier werden „Inneres und Äußeres“ nicht als getrennte Bereiche, sondern „als Momente eines Strukturierungsprozesses verstanden ..., in dem sich Eigenes und Fremdes wechselseitig relativieren und bestimmen". Die zugrunde liegende Ordnungsstruktur ist nicht mehr ambivalent, sondern polyvalent, eine „komplementäre Ordnung wechselseitiger Fremdheit". Und Schäfter verweist darauf, dass wirklich Fremdartiges sich auch bei bestem Willen nicht verstehen lässt und die innere Verarbeitungsfähigkeit schnell überfordert wird, womit es externe Bereiche gibt, „die prinzipiell nicht aneignungsfähig sind und daher ... in ihrem autonomen Eigenwert respektiert werden müssen". 
In diesem „Deutungsmuster komplementärer Fremdheit“ verlocken Schwellenerfahrungen nicht zu einer umfassenden „Ausweitung des Innen“, sondern werden als Zwang zur Anerkennung einer radikalen gegenseitigen Differenz, als Sensibilität für gegenseitige Fremdheit ,aufgefasst“. Fremdheit wirkt in einem solchen Bedeutungszusammenhang als Ferment einer (inter-)kulturellen und innerpsychischen Dynamik. „Da Fremdheit den ,blinden Fleck“ der eigenen Wahrnehmungsfähigkeit erkennbar“ macht, wird sie „so zur mühevollen Erfahrung einer gegenseitigen Grenze“. Daraus könnten nach Schäfter neue Formen von „Gemeinsamkeit“ entstehen, „die sich als tragfähiger erweisen, als die Einfühlung in vermeintlich , universelle“ Grundlagen des Humanen" (SCHÄFFTER 1997, 19-23).

\subsection{Exkurs „Kulturelle Selbstreflexion“}

„Unter bestimmten Bedingungen kann interkulturelles Lernen ... Fremdenfeindlichkeit vergrößern“" (NeSTVOGEL 1988, 48). Der Versuch allen Ansprüchen gerecht zu werden - ein besonderer Mitmensch, ein beispielhafter Freund von Migranten und Migrantinnen sowie kultureller Vielfalt sein zu müssen (Ich darf keine Vorurteile haben! Ich darf kein Rassist sein! Ich darf nicht eurozentrisch sein! Ich darf kein Nationalist sein! usw.), ist eine - normative - Überforderung; er kann Schuldgefühle erzeugen und unterdrücken, kann Angst fördern und überspielen. Es mag verständlich sein, dass Menschen an Vielem ihres kulturellen Erbes nicht teilhaben wollen, es gern ausgrenzen, ja abspalten möchten:

- „Vielleicht will ich ein ,anderer“ Mann sein, und doch habe ich Anteil an der patriarchalen Unterdrückungsgeschichte der Frauen“ (ORTH 1989, 20);

- vielleicht bemühe ich mich, um das ,andere“ Deutschland, Österreich, Ungarn, und habe doch Anteil an der jeweiligen Geschichte;

- vielleicht bemühe ich mich um mehr internationale Gerechtigkeit, und habe doch Anteil an der Ungerechtigkeit dieser Welt.

Nicht nur Interkulturelles Lernen benötigt eine ,interkulturelle Selbstreflexion“, die zum Beispiel abendländische Höherwertigkeitsvorstellungen bewusst macht, Bilder und Assoziationen interkultureller Höherwertigkeitsvorstellungen aufspürt (,Ist Mitleid die vornehmste Form der Verachtung?") und intrakulturelle Höherwertigkeitsvorstellungen bewusst macht, da „sich Vorstellungen von der eigenen Höherwertigkeit nicht nur im Umgang mit Fremden zeigen, sondern auch im Umgang mit den eigenen Landsleuten“" (NESTVOGEL 1988, 44).

Renate NeSTVOGEL verweist aber auch darauf, dass kulturelle Selbstreflexion einen enormen Anforderungsdruck gerade auf die Wohlmeinenden ausüben kann. „Die Ängste werden dadurch vergrößert, dass ich wenig darüber weiß, welche Vorurteile, Klischeebilder unter anderem ich verinnerlicht habe. Wenn ich in solchen Situationen Verinnerlichtes ,veräußere', könnte es sein, dass ich mich entblöße“ $(1988,48)$. Mit der Angst und den aufkommenden Schuldgefühlen wächst die Abwehr. Nestvogel gibt daher den Rat, sich in der eigenen ethnozentrischen Begrenztheit zu erkennen und anzunehmen. In Anlehnung an E. Jouhny schlägt sie vor: 
Ich bin in eine bestimmte Gesellschaft hineingeboren, habe in dieser Gesellschaft bestimmte Normen und Werte, Selbst- und Fremdbilder erworben und verinnerlicht, und diese prägen mein Einstellungs- und Verhaltensmuster. Ich kann nichts dafür, dass ich Angehöriger einer herrschenden Mehrheit bin. Aber - und hier beginnt meine Arbeit - ich kann keineswegs davon ausgehen, dass ausgerechnet ich mit meinen Persönlichkeitsstrukturen frei von Geschichte bin, die sich über Generationen hinweg tradiert hat, und hier liegt meine Verantwortung: Nicht im Tragen von Schuldgefühlen, was mir ein Gefühl der Hilflosigkeit und Ohnmacht und damit Handlungsunfähigkeit vermittlen würde, sondern im Annehmen meiner ethnozentrischen Geprägheit und Begrenztheit und im Aufarbeiten derselben.

(NeSTVOGEL 1988, 48)

Fremde können uns nach SIMPFENDÖRFER (1981) zum Bewusstsein unserer kulturellen Ambivalenz verhelfen. Könnte ich die Angst vor mir selber verlieren und könnte dementsprechend die Angst vor dem Fremden weichen? Nimmt der Fremde mir gar die Angst vor mir, insofern er zu meiner Selbstaufklärung beiträgt? Aber die Ambivalenz bleibt doch, und die Realität menschlicher Begrenztheit wäre jedenfalls zu beachten.

\section{Kultur, Interkulturalität, Interkulturelle Kommunikation}

\subsection{Verständnis von Kultur}

Von der allen Menschen gemeinsamen Natur und den damit verbundenen Grundbedürfnissen zu unterscheiden ist ihre jeweilige Kultur, womit die Lebensform im umfassendsten Sinn gemeint ist. Es geht hier also nicht um Kultur als einem gesellschaftlichen Teilsystem, sondern um ein (Lebens-)Wissen, das eine Gruppe von Menschen teilt, in bestimmten Formen ausdrückt und darin zugleich weitergibt. Auf Kultur als Lebensform sind Menschen angewiesen, um aufwachsen zu können. Sie eignen sich die jeweilige Kultur an, interpretieren sie aber auch individuell.

Heringer schlägt vor, in der Kultur eine Lebensform zu sehen und sie als „Objekt besonderer Art" zu betrachten, als „eine menschliche Institution, die auf gemeinsamem Wissen basiert". Entstanden in gemeinsamem menschlichen Handeln, wurde sie nicht gewollt, sondern ,ist vielmehr ein Produkt der Unsichtbaren Hand“, ,ein Potenzial für gemeinsames sinnträchtiges Handeln“. Dieses „Potenzial zeigt sich nur in der Performanz, im Vollzug“ und ,ist entstanden über Performanz“ (HERINGER 2004, 107).

Kultur erfassen bedeutet stets auch Bedeutungen erschließen. Angesichts der Vielfalt an Kulturen sind kulturelle Differenzen wahrzunehmen und anzuerkennen, wobei es gilt, dabei kulturelle Zuschreibungen und Typisierungen zu vermeiden. Falls sie auf abstrakter Ebene ihre Gültigkeit haben, treffen sie doch nicht die konkrete Kommunikationssituation. Außerdem ist Kultur nie etwas Abgeschlossenes, sondern unterliegt einem ständigen, mehr oder weniger starken beziehungsweise schnellen 
Wandel, ist flexibel und nichts Homogenes. Zugleich können kulturelle Differenzen tiefgreifender sein, als auf den ersten Blick angenommen (HERINGER 2004, 158-59). Auch für Auernheimer dürfe, darüber wäre seiner Meinung nach Konsens im Diskurs um interkulturelle Erziehung, ,Kultur nicht als statisches, homogenes, hermetisch abgeschlossenes System betrachten werden“. Und Hierdeis stellt fest, dass für „die mit dem Zusammenhang von Fremdheit und Kultur befassten Wissenschaften ... . heute ein dynamischer, offener, pluraler Kulturbegriff selbstverständlich" ist (HIERDEIS 2005, 127). Ähnlich kritisiert WELSCH (1992) in seiner Argumentation für Transkulturalität das Konzept einer homogen und abgegrenzten, abgrenzbar gedachten Kultur, weil die Grenzen zwischen fremd und eigen durchlässig geworden sind. Auch wenn man seine Position für Transkulturalität nicht teilt, muss man sich doch der Frage stellen, ob es nicht eine ideologische Position darstellt, in der Kultur statisch gedacht wird (mit allen gesellschaftlichen und politischen Konsequenzen), die Exklusion legitimiert und jede Veränderung sanktioniert? In der geschichtlichen Realität, die auch aus einem längeren Zeithorizont zu betrachten ist, sind Kulturen doch von Veränderung und vom gegenseitigen Austausch bestimmt, was natürlich nicht Beliebigkeit und Austauschbarkeit bedeutet.

\subsection{Interkulturalität}

Schlüsselfragen im Bereich der Interkulturalität, die zugleich zentrale Forschungsfragen sind, hat Hierdeis gesammelt, wie zum Beispiel:

_ „Wie lässt sich Interkulturalität als Prinzip kulturbewussten Mitdenkens des Anderen und Fremden verstehen?"“

_ „Wie funktioniert Interkulturalität als Modus kooperativer Selbstaufklärung und wissenschaftlicher Partnerschaft?"

- „Wie lassen sich die interkulturellen Verstehensprozesse aus den Grenzen der reinen Sprachlichkeit befreien?"

- „Worin genau bestehen Kulturunterschiede?“(HIERDEIS 2005, 129-30).

\subsection{Interkulturelle Kommunikation}

Versteht man Interkulturelle Kommunikation als Kommunikation von Menschen, die sich auf ihre je verschiedenen Kulturen als unterschiedliche Sinn- und Deutungssysteme beziehen, dann ist die Notwendigkeit der dann besonderen Verständigungsarbeit nachzuvollziehen, die im Falle einer intrakulturellen Kommunikation in dieser Weise nicht erforderlich scheint. Damit wird weder eine Kommunikation von Kulturen behauptet, noch Kultur als geschlossenes System oder als homogen verstanden. Interkulturelle Kommunikation versteht sich als Lernprozess (HIERDEIS 2005, 135), der mit besonderen Kommunikationsbarrieren verbunden ist. Hinzu kommt noch ein in der Regel kaum bedachter Aspekt: Besonders Migranten und Migrantinnen befinden sich oft in Kommunikationssituationen, die sich im Kontext von Institutionen mit dem An- 
spruch der Standardisierung und Normalisierung ereignen. Dieser institutionelle und strukturelle Kontext beeinflusst die Kommunikationssituation nachhaltig. Nun sind Bemühungen um eine kreative Bewältigung von Kommunikationsbarrieren in der Interkulturellen Kommunikation zum Scheitern verurteilt, wenn der Kontext darauf angelegt ist, diese Barrieren zu erhöhen. Bereits vor über zwei Jahrzehnten hat Radtke darauf aufmerksam gemacht, dass pädagogische Bemühungen um das interkulturelle Lernen ins Leere gehen, wenn der geheime Stundenplan unverändert auf Diskriminierung setzt. Diese Kritik wurde weiterentwickelt zum Begriff der „Institutionellen Diskrimierung" (GOMOLLA \& RADTKE 2002).

\section{Interkulturelle Kompetenz}

Eine differenzierte Auseinandersetzung zum Verständnis von Interkultureller Kompetenz hat AUERNHEIMER (2002) gefördert. Obwohl das Konzept einer Interkulturellen Kompetenz umstritten ist und unter Kulturalismusverdacht steht, ist es zu einer fast selbstverständlichen Forderung und Agenda im Bereich der Sozialarbeit und Bildung geworden. Konsens besteht unter den Befürwortern, dass Interkulturelle Kompetenz Teil des Professionalitätsverständnisses sein muss, womit auch Konsequenzen für die Ausbildung verbunden wären. Die Diskussion rezipierend wird „Interkulturelle Verständigung als Haltung, Prozess und methodisches Instrumentarium“ verstanden, „, die in den Kontext dieser umfassenden Wandlungsprozesse eingebettet sind und ihnen gegenüber ihre Wirksamkeit beweisen müssen“ (HANDSCHUCK \& KLAWE 2004, 27). Im Anschluss an Kleve wird ,an die Stelle des Handlungsparadigmas einer Differenzminimierung eine Grundhaltung der Differenzakzeptanz" gesetzt (HANDSCHUCK \& KLAWE 2004, 35).

Die besondere kulturelle Lebensweise jeder Gruppe und jedes Milieus einer Gesellschaft folgt einer eigenen „Landkarte der Bedeutung“ (LUTZ 1992), mit deren Hilfe die Welt für ihre Mitglieder verstehbar wird, aber zugleich auch einem Veränderungsprozess unterliegt. Der Interkulturellen Verständigung geht es nicht um Machtgewinn über andere - durch Informationsvorsprung oder Deutungshoheit - sondern darum, diese „Landkarten der Bedeutung“ innerhalb kommunikativer und reflexiver Prozesse, Prozessen zwischen Gleichen und Unterschiedlichen, zu entschlüsseln mit dem Ziel der Anerkennung. Die zu oft feststellbare Ethnisierung und Selbstethnisierung können als Versuche verstanden werden, in der gesellschaftlichen Unübersichtlichkeit durch eine klare Grenzziehung zwischen ,wir" und ,ihr“ wieder Überblick zu gewinnen. Der Verlust der Gestaltungsmacht wird durch Definitionsmacht wettgemacht, die Abwertung der „Anderen“ dient der Aufwertung der „Eigenen“.

Die Vermittlung von Orientierungswissen und konkrete Begegnungssituationen können unter Umständen hilfreiche Elemente bei der Vermittlung Interkultureller Kompetenz sein, aber Psychoanalyse und Ethnopsychologie verweisen darauf, dass der Umgang mit Fremden wesentlich stärker von der jeweiligen Person und ihrer je 
eigenen Persönlichkeitsstruktur als vom Verhalten und der kulturellen Differenz der Fremden abhängig ist. Erforderlich ist unter anderem:

- die eigene Haltung reflektieren und falls notwendig verändern;

- ein Gespür für die eigene Begrenztheit und Subjektivität entwickeln;

- die Wahrnehmung von Unterschieden, Widersprüchen sowie die eigenen Deutungsmuster des Fremden erweitern.

Interkulturelle Kompetenz als Schlüsselkompetenz angesichts von Globalisierung, Migration, Pluralisierungen der Lebenswelten, Identitätsdiffusion, Risikogesellschaft, et cetera kann verstanden werden als

die Fähigkeit professioneller Akteure, eine Balance zwischen einem akzeptierenden, dialogischen Umgang mit Differenz und einer Orientierung an universellen Menschenrechten und dem Recht auf Teilhabe herzustellen.

(HANDSCHUCK \& KLAWE 2004, 46)

Dazu gehören allgemeine soziale und kommunikative Kompetenzen wie auch etwa die Fähigkeit zur Perspektivenübernahme und migrationsspezifisches Wissen, womit nicht primär „Faktenwissen“, sondern „Deutungswissen“ gemeint ist.

\section{Aktuelle Ausbildungsangebote}

\subsection{Kursangebot „Virtuelle Hochschule Bayern“}

An der „Virtuellen Hochschule Bayern“ (www.vhb.org) wird ein Kurs „Grundlagen der Interkulturellen Kommunikation" von Heringer (Augsburg) angeboten. Dieses virtuelle Seminar besteht aus drei Submodulen. Das erste Submodul „Kultur erfassen“ leitet zur Auseinandersetzung mit den zahlreichen Definitionsversuche zum Thema Kultur an und fragt nach Methoden, die imstande wären, kulturelle Differenzen erfassen zu können. Das zweite Submodul „Kulturstandards“ beleuchtet kritisch die Versuche, Kultur über Kulturstandards und Stereotypen zu erfassen. Das dritte Submodul „Kultur und Sprache“ öffnet den Blick auf den Zusammenhang von Sprache und Kultur, der im Bereich von „Hotspots“, „Hotwords“ und „Somatismen“" vermittelt wird und auch nonverbale Sprache einschließt.

„Interkulturelle Kompetenz“ ist ein weiterer Kurs, von Moosmüller (München) verantwortet, dessen Lernziele sind:

- Relativierung der eigenen ethnozentrischen Haltungen und Einstellungen,

- verschiedene kulturelle Perspektiven einnehmen können,

- Entwicklung von Kategorien zur Erfassung von kultureller Differenz,

- Erleben und Verstehen der Wirkweisen von Stereotypen und Vorurteilen sowie von Diskriminierung und Rassismus,

- Aufzeigen von Möglichkeiten Interkultureller Kompetenzentwicklung. 


\subsection{Semmelweis Universität Budapest}

Am Institut für Mentalhygiene der Semmelweis Universität wird ein Masterstudium vorbereitet, das auch ein Modul zu Interkultureller Kommunikation enthält. Die Blocklehrveranstaltung „Interkulturelle Kommunikation“ dient durch die Einführung in verschiedene theoretische Ansätze und Anregungen zur Reflexion der eigenen Person und Praxis der angemessenen Bewältigung von Situationen Interkultureller Kommunikation im Kontext des Berufsfeldes und somit dem berufsspezifischen Aufbau von Interkultureller Kompetenz. Die Lehrveranstaltung verfolgt im Einzelnen folgende Ziele:

- Wahrnehmungsmuster von Alterität und Alienität reflektieren;

- mit kultureller Selbstreflexion nach E. Jouhny vertraut werden;

- Fremdheit als Ressource erkennen;

- Interkulturelle Kompetenz im Rahmen des Berufsfeldes erwerben.

Diesen Zielen entsprechen folgende Inhalte:

- Konstruktion und Verflechtung von Alterität und Alienität;

- kulturelle Kommunikation und Identität;

- Erfahrungsweisen von Fremdheit nach Ortfried Schäffter,

- Prozess der Konstruktion von Bildern (Identifikation und Projektion) nach Daniel Frei;

- Interkulturelle Kommunikation am Beispiel berufsfeldspezifischer Fallstudien.

\section{Referenzen}

AUERnHEIMER, G., Hrsg. (2002) Interkulturelle Kompetenz und pädagogische Professionalität (Opladen: Leske \& Budrich).

BEYER, P. (1994) Religion and Globalization (London: Sage).

Eine Verfassung für Europa (2005) heruntergeladen am 10. Jul 2005 von http://europa.eu.int/ constitution/de.

FRITSCH-OPPERMANN, S.C. (2005) 'Globalisierung als Bedingung interreligiösen Lernens' in P. SCHREINER, U. SIEG \& V. ELSENBAST, Hrsg., Handbuch Interreligiöses Lernen (Gütersloh: Güterloher).

Gomolla, O. \& F.-O. RADTKE (2002) Institutionelle Diskriminierung: Die Herstellung ethnischer Differenz in der Schule (Opladen: Leske \& Budrich).

HANDSCHUCK, S. \& W. KLAWE (2004) Interkulturelle Verständigung in der Sozialen Arbeit: Ein Erfahrungs-, Lern- und Übungsprogramm zum Erwerb interkultureller Kompetenz (Weinheim \& München: Juventa).

HERINGER, H.J. (2004) Interkulturelle Kommunikation: Grundlagen und Konzepte (Tübingen $\&$ Basel: Francke).

HIERDEIS, H. (2005) Fremdheit als Ressource: Probleme und Chancen Interkultureller Kommunikation: Ein Studien- und Arbeitsbuch (Innsbruck: Studia). 
HolzBrecher, A. (1997) Wahrnehmung des Anderen: Zur Didaktik interkulturellen Lernens (Opladen: Leske \& Budrich).

Kirchenamt der EKD, Hrsg. (2003) Maße des Menschlichen: Evangelische Perspektiven zur Bildung in der Wissens- und Lerngesellschaft: Eine Denkschrift des Rates der Evangelischen Kirche in Deutschland (Gütersloh: Gütersloher).

LÖFFELHOLZ, M. \& A. HEPP (2002) 'Transkulturelle Kommunikation: Einführung in die Grundlagentexte' in A. HEPP \& M. LÖFFELHOLZ, Hrsg., Grundlagentexte zur transkulturellen Kommunikation (Konstanz: UVK) 11-33.

LuTZ, H. (1992) 'Ist Kultur Schicksal? Über die gesellschaftliche Konstruktion von Kultur und Migration' in R. LEIPRECHT, Hrsg., Unter Anderen - Rassismus und Jugendarbeit (Duisburg: DISS) 43-62.

MaletzKe, G. (1966) 'Interkulturelle Kommunikation und Publizistikwissenschaft', Publizistik $11,318-31$.

NeIDleIn, H.-C., Hrsg. (2004) Bericht über die menschliche Entwicklung 2004: Kulturelle Freiheit in unserer Welt der Vielfalt (Berlin: UNO).

Nestvogel, R. (1988) 'Kann die Aufrechterhaltung einer unreflektierten Mehrheitskultur die Aufgabe der Pädagogik sein?', Zeitschrift für Pädagogik 39, 23. Beiheft, 41-49.

Novy, A. (2005) 'Menschen machen Räume: Zum unglaublichen Bedeutungsverlust der Regionen in der Globalisierungsdebatte' in G. FASCHINGEDER \& N. ORNIG, Hrsg., Globalisierung ent-wickeln: Eine Reflexion über Entwicklung, Globalisierung und Repolitisierung (Wien: Mandelbaum) 109-12.

ORTH, G. (1989) ,Überlegungen zur Praxis interkulturellen Lernens‘, Zeitschrift Entwicklungspädagogik 12, 20.

PEuKert, H. (2004) ,Bildung und Religion: Reflexion zu einem bildungstheoretischen Religionsbegriff' in K. Dethloff, R. LAngthaler, H. Nagl-DoceKal \& F. WOLfRAm, Hrsg., Orte der Religion im philosophischen Diskurs der Gegenwart (Berlin: Parerga) 363-86.

Robertson, R. (1992) Globalization: Social Theory and Global Culture (London: Sage).

Robertson, R. (1998) 'Glokalisierung: Homogenität und Heterogenität in Raum und Zeit' in U. BECK, Hrsg., Perspektiven der Weltgesellschaft (Frankfurt a.M.: Suhrkamp) 192-220.

SCHÄFFTER, O. (1997) 'Modi des Fremderlebens: Deutungsmuster im Umgang mit Fremdheit' in O. SCHÄFFTER, Das Eigene und das Fremde: Lernen zwischen Erfahrungswelten: Aufsätze zu einer Theorie der Fremderfahrung (Berlin: Humboldt-Universität) 1-26.

SCHREITER, R. J. (1997) Die neue Katholizität: Globalisierung und die Theologie (Frankfurt a.M.: IKO).

SIMPFENDÖRFER, W. (1981) "„Sich einleben in den größeren Haushalt der bewohnten Erde“: Ökumenisches und ökologisches Lernen' in H. DAUBER \& W. SIMPFENDÖRFER, Hrsg., Eigener Haushalt und bewohnter Erdkreis (Wuppertal: Jugenddienst) 64-93.

Watzlawick, P., J. BEAVIN \& D.D. JaCKSON (1969) Menschliche Kommunikation: Formen, Störungen, Paradoxien (Bern: Huber).

Welsch, W. (1992) ,Transkulturalität: Lebensformen nach der Auflösung der Kulturen', Information Philosophie 20, 5-20. 